\title{
La comprensión de textos académicos en un contexto cooperativo guiado a través de la enseñanza recíproca
}

\author{
Salvador Santiago Pedro
}

UNAM. Facultad de Psicología, México

\section{México}

santiagopesa@hotmail.com 


\section{Resumen}

Introducción. En el presente trabajo se aplicó un programa de intervención educativa, diseñado con el fin de favorecer el desarrollo de estrategias en la comprensión de textos académicos, utilizando textos expositivos. El programa se desarrolló en un contexto cooperativo ya que ofrece la oportunidad de compartir experiencias y desarrollar una interacción social entre los compañeros. La estructura cooperativa fue guiada a través de la enseñanza recíproca con el fin de promover la autonomía del aprendizaje.

Método. Se realizó el estudio experimental con estudiantes de segundo semestre, en una Preparatoria incorporada a la Universidad Autónoma del Estado de México. Las sesiones de la investigación se realizaron en los salones de clases de dos horas diarias. El cuestionario diagnóstico de la forma de estudiar del alumno aplicado como pre-test y pos-test es una adaptación del inventario de auto-reporte de estrategias de aprendizaje (EDA) y el seguimiento de la práctica-guiada a través de la enseñanza recíproca con textos expositivos (Geografía y Ética) de las materias que se imparten en la institución.

Resultados. Los resultados de esta investigación indican que los alumnos que participaron en el taller incrementaron sus habilidades en el uso de estrategias de aprendizaje en el momento de trabajar un texto de estructura expositiva. en un contexto cooperativo mediante la prácticaguiada de la enseñanza recíproca

Discusión. Este trabajo confirma la necesidad de instrumentar programas educativos que permitan desarrollar en los estudiantes de nivel medio superior la adquisición de nuevos conocimientos mediante la comprensión de textos académicos, así como promover estrategias de aprendizaje que regulen su propio aprendizaje.

Palabras Clave: Estrategias de Aprendizaje, Enseñanza Recíproca, Comprensión de Textos, Textos Expositivos. 


\section{Introducción}

La investigación y el desarrollo de modelos de intervención se basan en la premisa de que la capacidad del alumno para adquirir y utilizar la información contenida en textos académicos, puede mejorarse mediante un entrenamiento en estrategias de procesamiento de la información apropiadas (Dansereau, 1985; citado por Díaz-Barriga, 1988). Las estrategias de aprendizaje pueden diferir en el sentido de su nivel de especialización o generalidad. Existen modelos de intervención centrados en el entrenamiento de estrategias de alto nivel o independientes del contenido, las cuales sirven para propósitos generales que pueden transferirse a un amplio rango de tareas de aprendizaje y tipos de textos académicos (DíazBarriga, 1988).

Pozo, (1990), Nisbet y Shucksmith (1987) definen a las estrategias de aprendizaje como los procesos que sirven de base a la verbalización de las tareas intelectuales, ya que son una serie de habilidades utilizadas con un determinado propósito que se realizan con el fin de facilitar la adquisición, el almacenamiento y la utilización de la información. Por su parte Monereo (1998), las define como procesos de toma de decisiones e intencionales, en los cuales el alumno elige y recupera de manera coordinada, los conocimientos que necesita para complementar una determinada demanda de un objetivo, dependiendo de las características de la situación educativa en que se produce la acción. Estos tipos de estrategias de enseñanza y aprendizaje se encuentran involucrados en la promoción de aprendizajes significativos a partir de los contenidos escolares.

De esta manera las estrategias no son más que simples secuencias de habilidades, que van más allá de las reglas o los hábitos que aconsejan algunos manuales sobre técnicas de estudio. Las estrategias representan habilidades de un orden más elevado que controlan y regulan las habilidades más específicamente referidas a las tareas o prácticas educativas. Son de una naturaleza general en la cual los alumnos realizan actividades como planificar o comprobar en diferentes situaciones o problemas.

La metacognición es de gran importancia en el proceso de aprendizaje ya que activa la participación del alumno en su proceso de aprendizaje. Como señala Flavell (1978, citado por Burón, 1993), la metacognición hace referencia al conocimiento de los propios procesos cognitivos, de los resultados de esos procesos y de cualquier aspecto que se relacione con ellos, es decir, a la continua observación de estos procesos en relación con los objetos 
cognitivos sobre los que se apoya, generalmente al servicio de alguna meta concreta $u$ objetivo.

Para que esta actividad metacognitiva responda a la capacidad que posee un alumno, implica que a través de la práctica el estudiante aprenda a examinar críticamente la materia de estudio, al hacerlo verifica, clarifica, amplia y/o reemplaza la información más precisa. De esta manera, la metacognición está en el plano de la conceptualización y en el de las abstracciones reflejadas, esto es lo que permite reflexionar sobre lo que se ha hecho, sobre el conocimiento que se tenia y, por consiguiente, llevar a cabo la autorregulación consciente. Así, lo que hace posible la metacognición son los esquemas conceptuales a los cuales las estrategias se encuentran integradas. De este modo, este proceso metacognitivo se realiza de manera integral, interactivo e independiente en el desarrollo-planificación, mantenimientoseguimiento y evaluación, como se menciona en las siguientes fases:

\section{Antes de la lectura. (EI lector desarrolla un plan de acción)}

- Establece metas para la lectura.

- Activa el conocimiento previo acerca del tópico a ser leído.

- Explora el material escrito para determinar la estructura del texto.

- Hace predicciones acerca de lo que aprenderá.

\section{Durante la lectura.}

- Aplicar un número disponible de estrategias.

- Resumir lo que ha leído.

- Reconocer el significado de las palabras y frases que no entiende completamente.

- Reconocer el vocabulario clave.

- Inferir conceptos y significados.

- Hacer preguntas que pueden ser respondidas al leer el texto.

- Darse cuenta cuando surgen problemas de comprensión.

- Evaluar las estrategias a ser utilizadas. 


\section{Después de la lectura (EI lector evalúa el plan de acción).}

- Revisar la actividad de lectura para la comprensión del contenido.

- Resumir las ideas clave del texto.

- Evaluar y reflejar que aprendió de la lectura.

- Pensar acerca de qué necesita para aprender más del tópico.

- Determinar las relaciones entre el tópico y otra información aprendida.

- Reflexionar acerca de las estrategias que mejor le sirvieron, cuáles menos y por qué.

- Releer el texto para ajustar predicciones, inferencias y decidir en qué aspecto requiere documentarse más. (Muriá, 1994; García, Riggs y Cañizales, 2001).

En este sentido, García, Riggs y Cañizales (2001) señalan que, el estudiante al interactuar en un contexto y con el texto activa su repertorio de estrategias de comprensión, esto es, la activación del conocimiento previo, determinación del significado de las palabras, establecimiento de diferencias a partir del texto y utilización de la estructura del texto para localizar la información. En cuanto al contexto dirige su propósito de la lectura y el desarrollo de un plan para lograr la tarea asignada y una comprensión básica del tipo de material. De esta manera, el lector hace ajustes basados en la identificación de patrones y el texto narrativo o expositivo que contribuirá a la comprensión y a la localización de importantes detalles.

La comprensión de textos se considera una actividad constructiva, porque durante este proceso el lector no realiza simplemente una transposición unidireccional de los mensajes comunicados en el texto a su base de conocimientos, equivale también a formarse una representación del contenido del mismo. El lector trata de construir una representación fidedigna a partir de los significados sugeridos por el texto, explotando los distintos índices y marcadores psicolingüísticos y los de formato que se encuentran en el discurso escrito. La construcción de esta representación es el resultado de un proceso interactivo en el que intervienen, tanto las características del texto como distintos tipos de conocimiento del sujeto (Díaz-Barriga y Hernández, 2002; Alonso y Carriedo, 1999).

Para lograr una comprensión adecuada del texto, es necesario el control y la regulación de los recursos cognitivos, saber de que forma activar los conocimientos y utilizarlos en un texto determinado. Para ello es necesario poseer procedimientos específicos o estratégicos y 
aplicarlos en forma propositiva y autorregulada (García Madruga et al, 1995). En este sentido, el lector que intenta comprender un texto y que desea "leer para aprender" debe planear el uso de distintos procedimientos estratégicos, los cuales también deben ponerse en marcha y supervisarse de manera continua, en función de un determinado propósito o demanda contextual planteada de antemano (Solé, 1992).

De acuerdo con Goodman (1986), el texto tiene una forma gráfica que posee dimensiones espaciales, tales como tamaño y direccionalidad. Además, todo texto escrito tiene una característica sintáctica, es decir, debe representar la sintaxis del lenguaje para que pueda ser comprensible. La puntuación, el orden de las oraciones, son índices que utilizan los lectores para llegar a la comprensión. Aznar, et al. (1991), mencionan que el texto tiene un valor de unidad comunicativa, tanto oral como escrito. Se considera a los textos como conjuntos verbales, que remiten al contexto en que han sido producidos. Por tanto, el texto es la unidad más grande en que pueden ser construidos las diferentes expresiones.

Todas las características del texto son utilizadas por el lector al hacer predicciones e inferencias en la construcción de significados. Van Dijk (1980), argumenta que un texto es abstracto, es un constructo teórico de varios componentes analizados en la gramática y en otros estudios discursivos. No sólo tiene una estructura gramatical, sino también estilística, retórica y esquemática. La importancia en la estructura del texto como una estrategia en la comprensión lectora, facilita la codificación de la información, la cual permite identificar las ideas principales del texto, organizándolas jerárquica y coherentemente, ayudándose de las claves textuales que indican la estructura de alto nivel del texto y del reconocimiento de la intención del autor, facilitando la elaboración de anticipación sobre el contenido, así como el recuerdo ya que los sujetos recuperan la información a partir de la estructura de alto nivel, siguiendo la búsqueda de los contenidos que dan apoyo a esta información (Meyer, citado por Vidal Abarca y Gilabert, 1991). La estructura de un texto hace referencia a como las ideas han sido organizadas conjuntamente, de manera que todo el contenido resulta de una cohesión, una coherencia global. En el texto debe existir una estructura que permita crear una coherencia referencial y causal entre las ideas expresadas en el texto. Esta coherencia referencial supone un solapamiento de argumentos entre las proposiciones que la integran y ello permite una continuidad en las ideas expresadas facilitando su comprensión (Ramírez y Velásquez, 1994). 
León y García Madruga (1991) proponen que la estructura del texto no sólo implica cómo las ideas han sido interrelacionadas en el pasaje, sino también el tipo de conexiones o relaciones que se establecen entre ellas, de modo que queden subordinadas unas a otras. La estructura del texto debe mostrar cómo las ideas centrales siguen la organización general mientras que otras ideas periféricas, subordinadas a ellas, inciden en aspectos secundarios o de detalle, complementando la información esencial. En los textos expositivos, la estructura del texto específica la conexión lógica entre ideas, así como la subordinación de unas ideas a otras, proporcionando patrones organizacionales que ayudan al lector a identificar y a unir las proposiciones relacionadas que son más importantes en el texto (Ambruster et al. ,1987).

Una de las metodologías más reconocida en la enseñanza de comprensión de textos es la enseñanza recíproca, que se refiere a un procedimiento instruccional que toma lugar en grupos de aprendizaje colaborativo y se caracteriza por una práctica guiada, respecto a la aplicación flexible de cuatro estrategias que son: hacer preguntas, resumir, clarificar y predecir. El maestro y el grupo toman turnos para guiar la discusión sobre el contenido del texto (Palincsar y Klenk, 1992). Para Brown y Palincsar (1989) la enseñanza recíproca se refiere a una serie de condiciones de aprendizaje en las que el alumno primero experimenta un conjunto particular de actividades cognoscitivas en la presencia de un experto (maestro) y asciende gradualmente a la ejecución de las funciones. Brown y Palincsar (1985), consideran a la enseñanza recíproca como una técnica instruccional para enseñar las cuatro estrategias (clarificación, cuestionamiento, resumen y predicción). La palabra "recíproca" (del latín "reciprocus") significa "ir hacía adelante y hacía atrás", o bien se refiere a un concepto relacional donde intervienen las partes en cuyo caso "una de las partes actúa o responde a algo previamente realizado por la otra parte”. Estas dos partes están representadas por el maestro y por el alumno. Ambas partes sostienen un diálogo acerca del mismo tema, el cual esta representado por el texto y comparte los mismos objetivos (metas).

Esta metodología, está en gran parte inspirada por las ideas de Vigotsky y sus principios teóricos los cuales son:

1. "El origen social de las funciones psicológicas superiores (éstos aparecen primero en el plano de lo interpersonal y después son internalizados y apropiados por el aprendiz).

2. La noción de Zona de desarrollo Próximo (ZDP) (la enseñanza recíproca fue diseñada para proveer una ZDP en las cuales los estudiantes, con la ayuda de los maestros y de 
compañeros más avanzados, toman una responsabilidad cada vez mayor en la realización de una actividad).

3. Los procesos psicológicos son adquiridos en una actividad contextualizada y holística. Las estrategias no son aprendidas fuera del contexto de la lectura funcional o significativa (Díaz-Barriga y Hernández, 2002, pp.300-301).

Rosenshine y Meister (1994, citado por Díaz-Barriga y Hernández, 2002), analizaron diferentes aplicaciones significativas desde que Brown y Palincsar las propusieron en los años sesenta. Estos autores encontraron dos modalidades de aplicación:

1. El uso exclusivo de la enseñanza recíproca, y

2. El empleo de la enseñanza recíproca precedido de series de enseñanza directa.

Las contribuciones que surgieron de Brown y Palincsar se describen a continuación (Rosenshine y Meister, 1994, citado por Suárez y Vásquez, 1997):

1. Modifican el papel del maestro, siendo éste quien apoya a los estudiantes a adquirir estrategias específicas para fomentar y monitorear su comprensión.

2. La identificación de cuatro estrategias específicas para fomentar y monitorear la comprensión.

3. La utilización de materiales de lectura actuales, ya que los materiales construidos específicamente no proveen un andamiaje que soporte los conocimientos de los estudiantes.

4. El refinamiento y popularización del concepto instruccional de andamiaje.

Las cuatro estrategias utilizadas durante la enseñanza recíproca fueron las siguientes (Brown y Palincsar, 1985):

Resumen. Es revisar el contenido de lo leído, requiere que el lector identifique e integre la información importante del texto, a partir de preguntarse ¿qué es lo que acabo de leer?, y exponerlo.

Clarificación. Es intentar resolver las comprensiones erróneas al enseñar a los lectores a permanecer atentos al vocabulario no familiar, referentes no claros, un texto 
desorganizado y conceptos difíciles; preguntándose, ¿me ha quedado claro?. En caso de ser necesario releer o buscar información adicional.

Cuestionamiento. Elaborar preguntas sobre el texto, como un medio de autoevaluación del conocimiento, mediante la siguiente cuestión, ¿qué preguntas podría hacerme el maestro de este material?.

Predicción. Es anticipar el posible desarrollo del texto futuro. Cuando el lector hace predicciones significativas activa su conocimiento previo relacionado con el texto $\mathrm{y} / \mathrm{o}$ su conocimiento sobre la estructura del texto. El lector puede hacerse la siguiente pregunta ¿qué es lo más probable que suceda a continuación en este texto?.

Palincsar y Klenk (1992), mencionan que las predicciones se basan en:

1. El conocimiento previo sobre el tópico leído.

2. Las claves provistas por el texto.

3. Las expectativas sobre lo que el autor abordará después.

En suma, se pretende ayudar al alumno a que construya su propio aprendizaje por medio de una instrucción que enriquezca sus esquemas de conocimiento, así como sus estrategias de comprensión de textos, haciendo de éste un proceso de comunicación, aprendiendo la lengua escrita en su uso e intercambio.

A través de este estudio se pretende comprobar si se favorece el desarrollo de estrategias de comprensión de textos empleando la práctica guiada a través de la enseñanza recíproca. El planteamiento de este estudio ha requerido:

A. Diseñar de un programa de entrenamiento en estrategias de comprensión de textos académicos en un contexto cooperativo, mediante la práctica-guiada a través de la enseñanza recíproca.

B. Aplicar el programa de entrenamiento en estrategias en la comprensión de textos académicos expositivos, en estudiantes de una Preparatoria Regional del Estado de México.

C. Observar el desempeño en la comprensión de textos académicos en un contexto cooperativo, mediante una práctica-guiada a través de la enseñanza recíproca en el aula. 
D. Evaluar la eficacia del programa en la comprensión de textos académicos en un contexto cooperativo a partir de la utilización de la enseñanza recíproca.

\section{Método}

\section{Participantes}

Participaron 60 estudiantes de segundo semestre, en una Preparatoria incorporada a la Universidad Autónoma del Estado de México. La selección se realizó mediante una muestra de tipo no probabilística, con sujetos voluntarios y se dividió en un grupo control de 30 alumnos y un grupo experimental de 30 alumnos.

\section{Procedimiento}

Se ha trabajado con las siguientes variables:

1. Comprensión de textos en los alumnos (Variable Dependiente). Explicación y ejecución de la tarea para la comprensión de los textos a partir de la aplicación de las estrategias de aprendizaje, regular cada una de las estrategias y elaborar conclusiones sobre los resultados y logros del taller.

2. Programa de entrenamiento, en la comprensión de textos académicos en un contexto cooperativo guiado a través de la enseñanza recíproca. (Variable Independiente) Conjunto de fases en las cuales se aplicó la enseñanza recíproca dentro del aula con alumnos de nivel medio superior en la comprensión de textos académicos.

Las fases del estudio realizado han sido:

1. Evaluación inicial: Durante esta fase participaron el grupo control y el grupo experimental. A los dos grupos se les aplicó un Pre-test que fue él Cuestionario Diagnóstico sobre la forma de Estudiar del Alumno, con la finalidad de realizar una evaluación acerca de la necesidad de programar un taller sobre estrategias en la comprensión de textos académicos y evaluar los conocimientos que poseían sobre este tema.

2. Programación del taller: De acuerdo a los resultados arrojados y las necesidades detectadas, se desarrollo el contenido del taller y el material necesario (textos expositivos, correspondientes a las materias de Geografía, y Ética) para su aplicación con el grupo experimental. 
3. Entrenamiento a los alumnos: En esta fase se desarrolló el taller "la comprensión de textos académicos en un contexto cooperativo de dos horas diarias a través de la prácticaguiada de la enseñanza recíproca.

4. Observación en la aplicación del taller en "la comprensión de textos académicos en un contexto cooperativo guiado a través de la enseñanza recíproca". La realización de esta tarea se llevó a cabo con el grupo experimental dentro del aula, con el fin de que los alumnos aplicaran los conocimientos y las habilidades adquiridas durante el taller.

\section{Análisis estadísticos}

Para realizar el análisis de datos obtenidos en las evaluaciones de la comprensión de textos académicos se empleó el análisis de varianza de Rangos de Friedman, ya que esta prueba no paramétrica es la más eficiente para medidas repetidas que provienen de la misma población. Se aplica cuando hay un nivel de medición ordinal y se trabaja con muestras pequeñas.

\section{Resultados}

Comparación Pre-test Grupo Control y Experimental.

En el cuestionario diagnóstico de la forma de estudiar de los alumnos se encontró lo siguiente:

Los resultados del pre-test, en el grupo experimental y el grupo control no se encontraron diferencias significativas en lo reportado en las cuatro categorías, como puede observarse en el gráfico 1.

Con respecto al grupo experimental en la categoría antes de la lectura se reportó un $34 \%$, en la categoría de metacognición obtuvieron un porcentaje de $31 \%$, en la categoría durante la lectura obtuvieron un porcentaje de $23 \%$ y en la categoría después de la lectura se obtuvieron porcentajes de $12 \%$. En cuanto al grupo control, los porcentajes que se obtuvieron por categoría fue el siguiente: Antes de la lectura 34\%, metacognición 31\%, durante la lectura $23 \%$ y después de la lectura $12 \%$. 
Gráfico 1. Porcentaje de alumnos del grupo experimental y el grupo control que responde en las cuatro categorías.

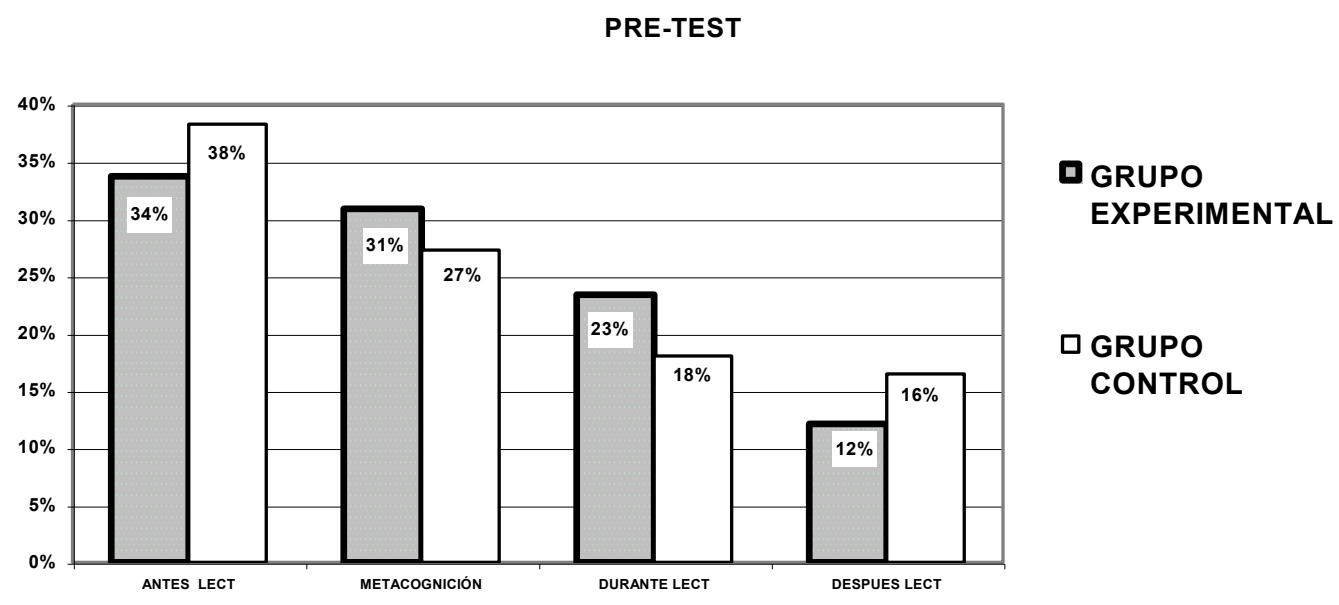

Análisis de los Efectos de la Enseñanza Recíproca.

Con respecto a la estrategia de clarificación (Gráfico 2), los alumnos del grupo experimental mostraron en lo general diferencias estadísticamente significativas en la estructura textual de Problema/ Solución $\left(\mathrm{x}^{2}=20.327, \mathrm{gl}=5, \mathrm{p}<.000\right)$.

\section{Gráfico 2. Medias de Rangos en la estrategia de Clarificación en el Grupo}

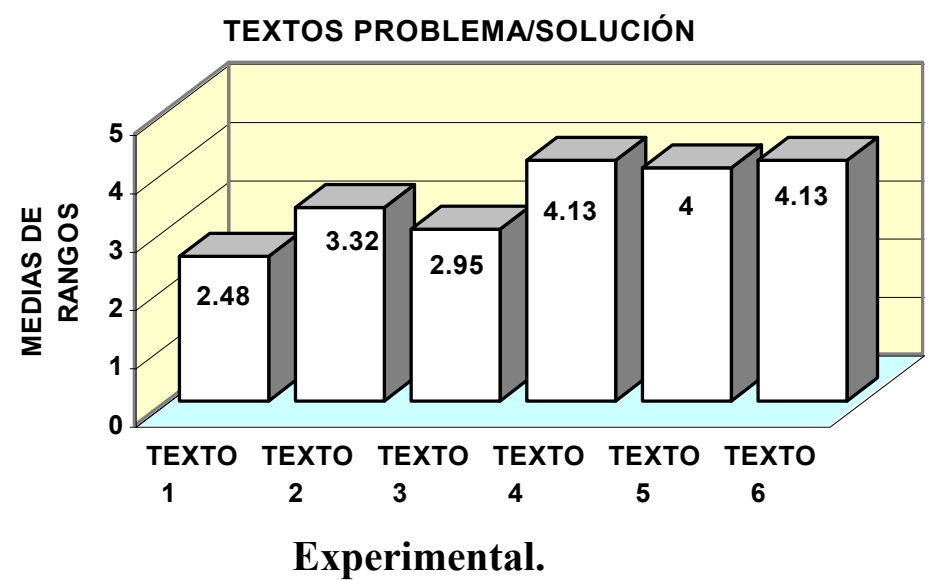

Como puede apreciarse en el gráfico 2, en la estrategia de clarificación se tuvo un incremento en la evolución del taller de comprensión de textos académicos, donde los 
alumnos fueron capaces de brindar una solución a un problema determinado, en la estructura textual de Problema/Solución. Con respecto al efecto en la estrategia de predicción (Gráfico 3) de los textos Problema/Solución $(x 2=32.07$, gl. $=5, \mathrm{p}<0.00)$.

Gráfico 3. Medias de Rangos en la Estrategia de Predicción en el Grupo Experimental.

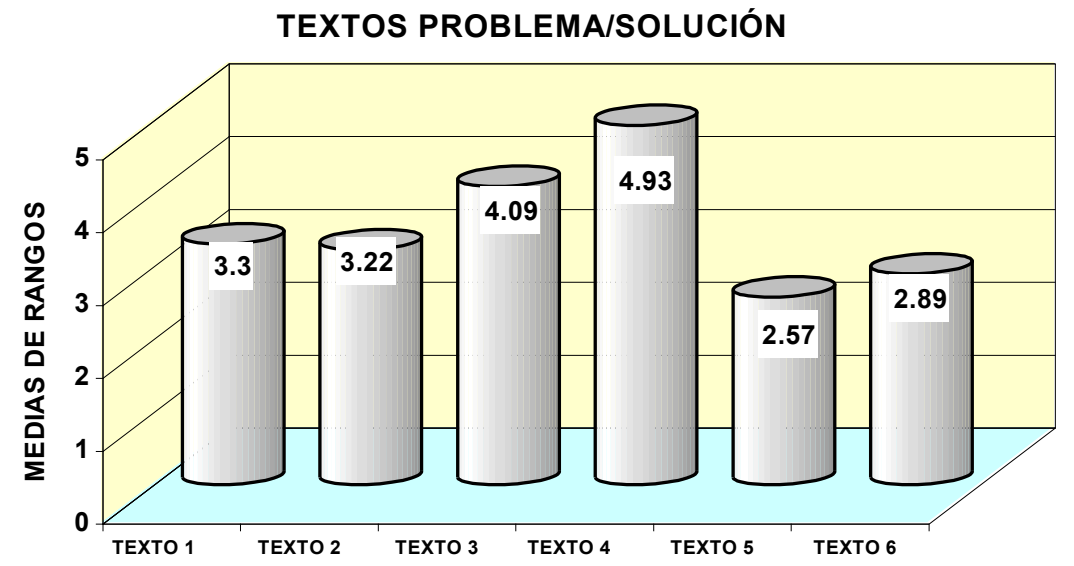

Como se puede apreciar en el gráfico 3, el puntaje que obtienen los alumnos en la comprensión de textos académicos es óptimo en la comprensión de la estructura textual Problema/Solución. En el análisis de la estrategia de cuestionamiento, se encontraron en lo general diferencias significativas (Gráfico 4) $(\mathrm{x} 2=20.32$, gl $=5, \mathrm{p}<0.00)$ en los textos de Problema/Solución.

Gráfico 4. Medias de Rangos en la estrategia de Cuestionamiento en el Grupo Experimental.

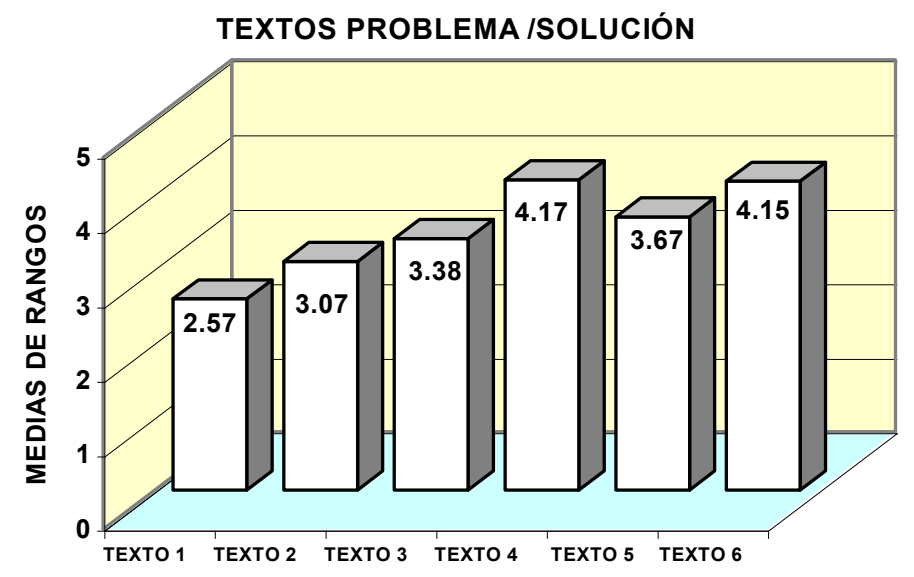


En el nivel de comprensión de los textos de Problema/Solución, los alumnos incrementaron su comprensión ante la presentación de un problema y determinar su posible solución.

En lo concerniente a la estrategia de Resumen, en los textos de Problema/Solución (Gráfico 5) se encontraron en general diferencias estadísticamente significativas (x2=92.66, $\mathrm{gl}=5, \mathrm{p}<0.00)$ en lo referente al taller de comprensión de textos académicos.

\section{Gráfico 5. Medias de Rangos en la estrategia de Resumen en el Grupo Experimental.}

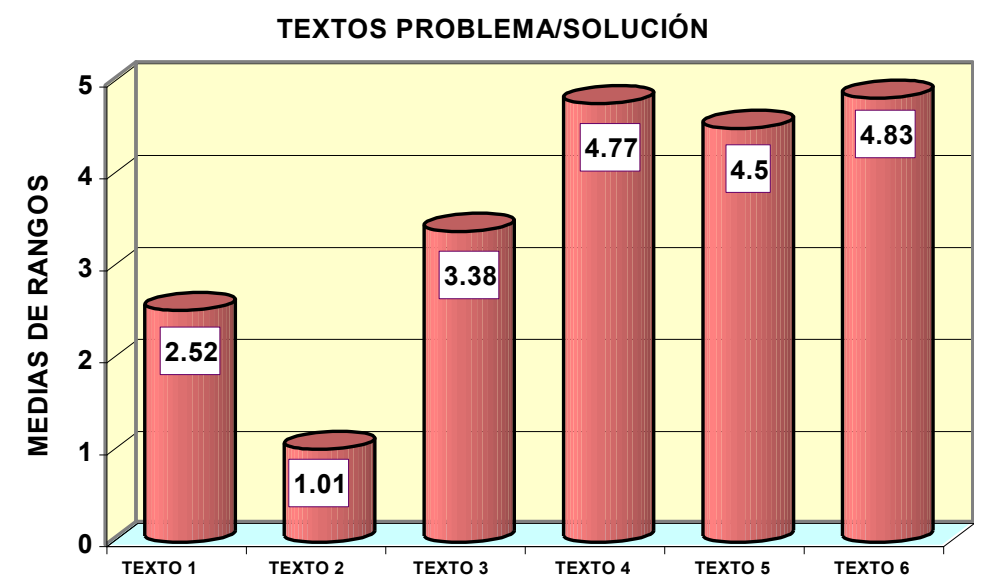

\section{Comparación Pos-Test Grupo Control Y Experimental.}

Con respecto, a lo indicado en la aplicación del pos-test el grupo experimental, reportó cambios significativos al obtener puntajes óptimos en tres categorías. En lo que respecta a la estrategia antes de la lectura se obtuvieron cambios significativos (ver gráfica 6), se observa un $3 \%$, en la estrategia durante la lectura se obtuvo un $25 \%$, así mismo, en la estrategia después de la lectura la puntuación obtenida fue de 35\% y finalmente en la estrategia de metacognición el puntaje fue de 37\%.

Por otro lado, se puede observar que el grupo control no reportó cambios significativos en las cuatro categorías en la aplicación pos-test, obteniendo puntajes, en las estrategias antes de la lectura $38 \%$, estrategias durante la lectura $25 \%$, estrategias después de la lectura $24 \%$ y metacognición 13\%, muy similares en la aplicación del pre-test. 


\section{Gráfico 6. Porcentaje de alumnos del grupo experimental y el grupo control que responde en las cuatro categorías.}

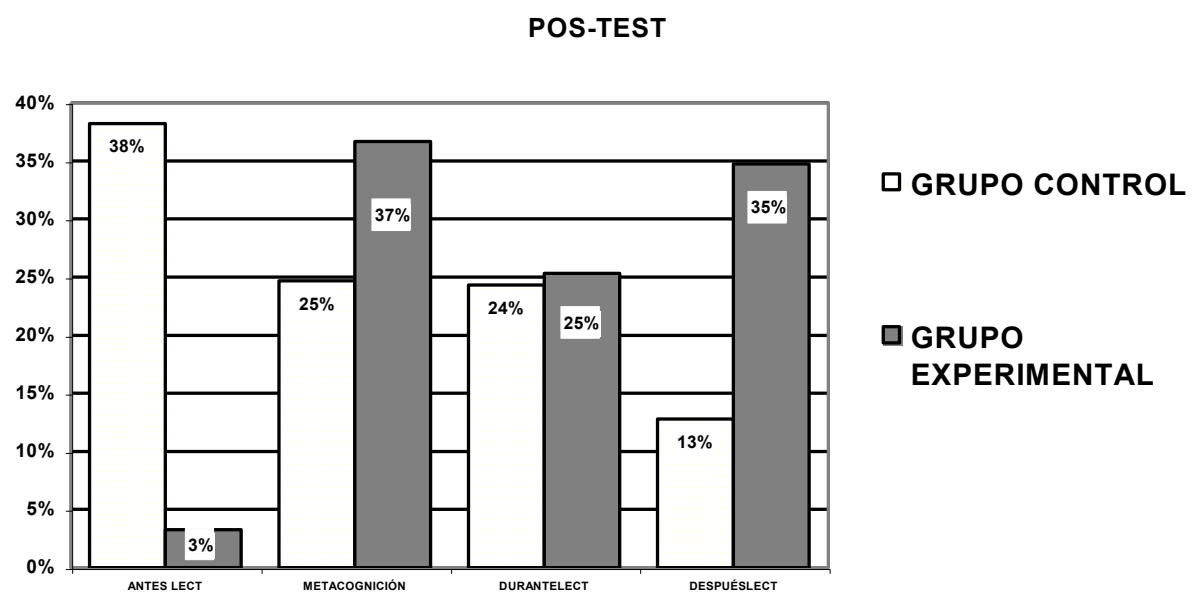

Con la finalidad de analizar la evolución de los alumnos en el taller de la comprensión de textos académicos, se presentan los resultados del análisis para cada una de las estructuras textuales, como se muestra en el anexo 1.

\section{Discusión}

A partir de la instrumentación del taller de trabajo se pudo observar que la enseñanza recíproca permite trabajar las cuatro estrategias (resumen, clarificación, cuestionamiento y predicción) de esta manera los alumnos guiaron la discusión sobre el contenido del texto para comprenderlo de forma conjunta.

Los resultados de esta investigación indican que los alumnos que participaron en el taller incrementaron sus habilidades en el uso de estrategias de aprendizaje en el momento de trabajar un texto de estructura expositiva. Ya que en el desarrollo del taller se promovieron las estrategias de aprendizaje en la comprensión de textos académicos en un contexto cooperativo mediante la práctica-guiada de la enseñanza recíproca, en los alumnos de nivel medio superior.

De los aspectos más sobresalientes se pudo observar que, existen diferencias en cuanto al rendimiento de cada uno de los alumnos y las estrategias de aprendizaje, situándolo en un nivel de enseñanza real, así como el efecto de las estructuras textuales que facilitaron su comprensión y el recuerdo de la información del contenido temático de cada texto que se evaluaba. Así, en el Cuestionario Diagnóstico, los resultados que se obtuvieron entre el grupo 
experimental y el grupo control en la aplicación del pretest, muestra que no existieron diferencias significativas en relación a lo reportado en cada una de las categorías del cuestionario (estrategias antes de la lectura, estrategias durante la lectura, estrategias después de la lectura y metacognición).

Por otra parte, en cuanto a lo reportado en el postest se observan diferencias significativas en tres de las cuatro categorías de evaluación en el grupo experimental (estrategias durante la lectura, después de la lectura y metacognición) en comparación con el grupo control que no reportó cambios en ninguna de las cuatro categorías de evaluación. Es importante señalar que la diferencia entre el grupo control y el grupo experimental en el postest fue en un orden de $35 \%$ en la categoría de antes de la lectura, lo que muestra que los alumnos del grupo control mantuvieron el mismo porcentaje que en el pretest. En cuanto a la siguiente categoría, durante la lectura, no se reportaron cambios significativos entre los grupos, ya que existe una diferencia del 1\%, mientras en la categoría después de la lectura el grupo experimental obtuvo diferencias significativas en un orden de $22 \%$ respecto al grupo control y en la categoría de metacognición se observa una diferencia significativa de $12 \%$, en este sentido se observó que los alumnos del grupo experimental presentaron un buen desempeño en el taller en cuanto a la aplicación adecuada de las estrategias de aprendizaje.

De acuerdo con los resultados del cuestionario diagnóstico, se puede afirmar que la aplicación y uso de estrategias de aprendizaje por parte de los estudiantes facilitan su desempeño en el aula. De esta manera, se promueve la adquisición de aprendizajes significativos.

Por otra parte, los datos obtenidos en la práctica-guiada a través de la enseñanza recíproca, revelan que se incrementó la comprensión de textos académicos en los alumnos del grupo experimental que participaron en el taller. En la estrategia de clarificación para los textos de Problema/Solución sé obtuvó un 96\% de alumnos que la realizaba adecuadamente, esto indica un aumento gradual en la comprensión de los textos expositivos en las sesiones de trabajo, es decir, que los alumnos son capaces de elaborar diferencias o semejanzas entre una o más temáticas, organizan la información y mantuvieron una mayor atención al momento de leer los textos que se les presentaron, ya que tenían que buscar información adicional o releer para obtener una mejor comprensión de lo leído.

Para la estrategia de predicción, los alumnos que realizaron adecuadamente esta tarea en el caso de los textos de Problema/Solución fue de un $76 \%$, lo que permitió que se 
obtuvieran diferencias significativas en este tipo de estructuras textuales. Esto puede indicar que el entrenamiento para predecir a través del titulo y después de la lectura fue efectivo de manera que los alumnos tienen un manejo adecuado de esta estrategia ya que para éstos es una herramienta útil y con resultados positivos. Además de que permite activar los conocimientos previos relacionados con los textos y generar expectativas sobre lo que el autor abordará después.

En el caso de la estrategia de cuestionamiento el 100\% alumnos obtuvieron puntuaciones estadísticamente significativas en las cuatro estructuras textuales. En este sentido, se puede considerar que el trabajo en esta estrategia permitió a los alumnos involucrarse activamente en la tarea de preguntar sobre las ideas principales o generar preguntas sobre un párrafo del texto, propiciando en los alumnos la regulación de su conocimiento y promoviendo un incremento en la comprensión de textos. Así, el alumno es capaz de formular preguntas sobre el texto y autoevaluar su conocimiento sobre lo leído.

Por otra parte, en la estrategia de resumen, el $86 \%$ de los alumnos obtuvieron puntuaciones estadísticamente significativas. Esto es, que el alumno integra e identifica la información más importante del texto. Por lo tanto, se puede considerar que el desarrollo del programa influyó para que los alumnos tuvieran un manejo de los elementos para generar ideas principales y evaluar la coherencia del texto. Estas mejoras se pueden atribuir, entre otros factores a la participación activa de los alumnos y la disposición de aprender significativamente los materiales y contenidos de aprendizaje durante el taller.

Este trabajo confirma la necesidad de instrumentar programas educativos que permitan desarrollar en los estudiantes de nivel medio superior la adquisición de nuevos conocimientos mediante la comprensión de textos académicos, así como promover estrategias de aprendizaje y que regulen su propio aprendizaje, es decir, que a través de la práctica los alumnos establezcan las diferencias a partir del texto y la utilización de la estructura del texto para localizar la información y construir significados. Además de fortalecer su habilidad como lector y aprendiz.

Promover con los alumnos habilidades estratégicas para la comprensión de textos, es permitir que los alumnos regulen su propio aprendizaje y desarrollen una actividad constructiva para generar nuevos conocimientos que sean significativos para obtener mejores resultados académicos. Es importante señalar, que se debe partir de lo que el alumno realmente sabe para generar zonas de desarrollo próximo a partir de un andamiaje tutor- 
aprendiz y gradualmente potenciar en el alumno la autorregulación de su propio aprendizaje. En el presente trabajo se observó que la enseñanza recíproca integra aspectos sociales e interactivos, que le permiten al alumno autorregular su aprendizaje y, por lo tanto, obtener mejores resultados académicos, también generar actividades de apoyo y estratégicas que lo motivan a aprender.

\section{Referencias}

Alonso, J. y Carriedo, N. (1999). Problemas de Comprensión Lectora: Evaluación e Intervención. En Monereo, C., y Solé, I. (Coord.), El asesoramiento Psicopedagógico: Una perspectiva profesional y constructiva. Madrid: Alianza.

Ambruster, D. F., Anderson, T. H. y Atertog, J. (1987). Does texts structures summarization instruction, facilitate learning from expository text. Reading Research Quarterly. 22 (35), 331-346.

Aznar, E., Cros, A. y Quintana, L. (1991). Coherencia textual y lectura. ICE. Barcelo na: Horsori.

Brown, A. y Palincsar, A. (1985). Enseñanza recíproca. Actividades para promover la lectura con la mente. (Trad. Hernández, G. y Velázquez, B. 1996). En Reading, Thinking and Concept Development. N. York: College Entrance Examination Board.

Brown, A. y Palincsar, A.(1989).Guided, Cooperative learning and individual knowledge Acquisition. En Resnick, L. (Eds.), Knowing, Learning and Instruction. Essays in honor of Robert Glaser. Lawrence Earlbawm, Publishers, A.S.A.

Burón, J. (1993). Enseñar a Aprender. Introducción a la metacognición. Bilbao, España: Mensajero.

Díaz-Barriga, F.(1988).Aprendizaje significativo y organizadores anticipados. Programa de Publicaciones de Material Didáctico, México:Facultad de Psicología, UNAM

Díaz-Barriga, F. Y Hernández, G. (2002). Estrategias docentes para un aprendizaje significativo: Una interpretación constructivista. México: Mc Graw Hill.

García Madruga, J. A., Martín, J. I., Luque, J.L. y Santamaría, C. (1995). Comprensión y adquisición de conocimientos a partir de textos. Madrid: Siglo XXI.

García, A; Riggs, E. Y Cañizales, R. (2001). Metacognición: punto de ignición del lector estrat lector estratégico. Lectura y Vida. 3, 28-35. 
Goodman, K. (1986). El proceso de lectura: Consideraciones a través de las lenguas y del desarrollo. En E. Ferreiro y M. Gómez Palacio (Comps.), Nuevas perspectivas sobre los procesos de lectura y escritura. México: Siglo XXI.

León, J. A. y García-Madruga, J. A. (1991). Memoria y Comprensión de textos. En J. M. Ruiz Vargas (Eds), Psicología de la memoria. España: Alianza Editorial.

Monereo, C. (1998).El asesoramiento en el ámbito de las estrategias de aprendizaje. En C. Monereo. (Coord.), Estrategias de Aprendizaje. Madrid: Visor.

Muriá, I. (1994). La enseñanza de las Estrategias de Aprendizaje y las habilidades metacognitivas. Perfiles Educativos, 65, 66-70.

Nisbet, J. y Schucksmith, J. (1987). Estrategias de aprendizaje. Madrid: Santillana.

Palincsar, A. y Klenk, L. (1992). Fostering literacy learning in supportive contexts (Trad. Hernández, G., 1996). Journal of learning Disabilities, 4 (4), 211-215.

Pozo, J. I. (1990). Estrategias de aprendizaje. En C. Coll; J. Palacios y A. Marchesi (eds.) Desarrollo psicológico y educación II. Psicología de la Educación. Madrid : Alianza.

Ramírez, L. y Velásquez, B. (1994). Un estudio comparativo sobre la comprensión de textos expositivos entre niños de 4to. y 6to. de primaria y de 2 do. de secundaria. Tesis de Licenciatura. Fac. Psicología: UNAM.

Solé, I. (1992). Estrategias de lectura. Barcelona: Graó-ICE.

Van Dijk, T. (1980). Estructuras y funciones del discurso. México: Siglo XXI.

Vidal-Abarca, E. y Gilabert, R. (1991). Comprender para aprender. Un programa para mejorar la comprensión y el aprendizaje de textos. Madrid: CEPE. 
ANEXO 1

CALIFICACIONES DE LA ENSEÑANZA RECÍPROCA DEL GRUPO EXPERIMENTAL

\begin{tabular}{|c|c|c|c|c|}
\hline $\begin{array}{l}\text { Estructura } \\
\text { Textual }\end{array}$ & $\begin{array}{l}\text { Estrategias de la } \\
\text { Enseñanza } \\
\text { recíproca }\end{array}$ & Textos & Medias & $\begin{array}{l}\text { Nivel de } \\
\text { significación }\end{array}$ \\
\hline \multirow{24}{*}{$\begin{array}{l}\text { PROBLEMA } \\
\text { / } \\
\text { SOLUCIÓN }\end{array}$} & \multirow{6}{*}{ Clarificación } & La Libertad & 2.48 & \multirow{6}{*}{$\mathbf{p}<.000$} \\
\hline & & El control de insectos & 3.32 & \\
\hline & & La Eutanasia & 2.95 & \\
\hline & & La contaminación Atmosférica & 4.13 & \\
\hline & & El Arroz & 4.11 & \\
\hline & & Normas de convivencia & 4.13 & \\
\hline & \multirow{6}{*}{ Predicción } & La Libertad & 3.32 & \multirow{6}{*}{$\mathrm{p}<.000$} \\
\hline & & El control de insectos & 3.22 & \\
\hline & & La Eutanasia & 4.09 & \\
\hline & & La contaminación Atmosférica & 4.93 & \\
\hline & & El Arroz & 2.57 & \\
\hline & & Normas de convivencia & 2.89 & \\
\hline & \multirow{6}{*}{ Cuestionamiento } & La Libertad & 2.57 & \multirow{6}{*}{$\mathrm{p}<.001$} \\
\hline & & El control de insectos & 3.07 & \\
\hline & & La Eutanasia & 3.38 & \\
\hline & & La contaminación Atmosférica & 4.17 & \\
\hline & & El Arroz & 3.67 & \\
\hline & & Normas de convivencia & 4.15 & \\
\hline & \multirow{6}{*}{ Resumen } & La Libertad & 2.52 & \multirow{6}{*}{$\mathbf{p}<.000$} \\
\hline & & El control de insectos & 1.01 & \\
\hline & & La Eutanasia & 3.38 & \\
\hline & & La contaminación Atmosférica & 4.77 & \\
\hline & & El Arroz & 4.55 & \\
\hline & & Normas de convivencia & 4.83 & \\
\hline
\end{tabular}

